\title{
Subgingival sites and oral cavity as reservoirs for Candida spp. in diabetics and non-diabetics
}

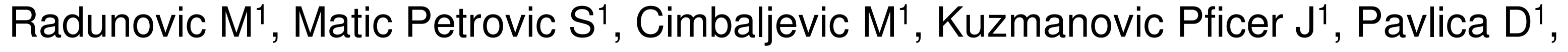

Arsic Arsenijevic V², Pucar $\mathrm{A}^{1}$

\section{School of Dental Medicine, University of Belgrade, Belgrade, Serbia \\ 2 School of Medicine, University of Belgrade, Belgrade, Serbia}

\section{Objectives}

Chronic periodontitis (CP) and oral mucocutaneous candidiasis have been considered as chronic complications of Type 2 Diabetes (T2D). The potential role of yeasts in the pathogenesis of periodontitis is especially important for diabetic patients. The aim of this study was to detect Candida spp. on the tongue and in subgingival sites from systematically healthy subjects and subjects with T2D and CP. Furthermore, the present study aimed to find a potential difference in the detection of yeasts on the tongue and in subgingival samples, in order to determine subgingival areas as potential reservoirs of yeasts.

\section{Methods}

A total of 146 patients were included in this study. They were divided into four groups as shown in figure 1.

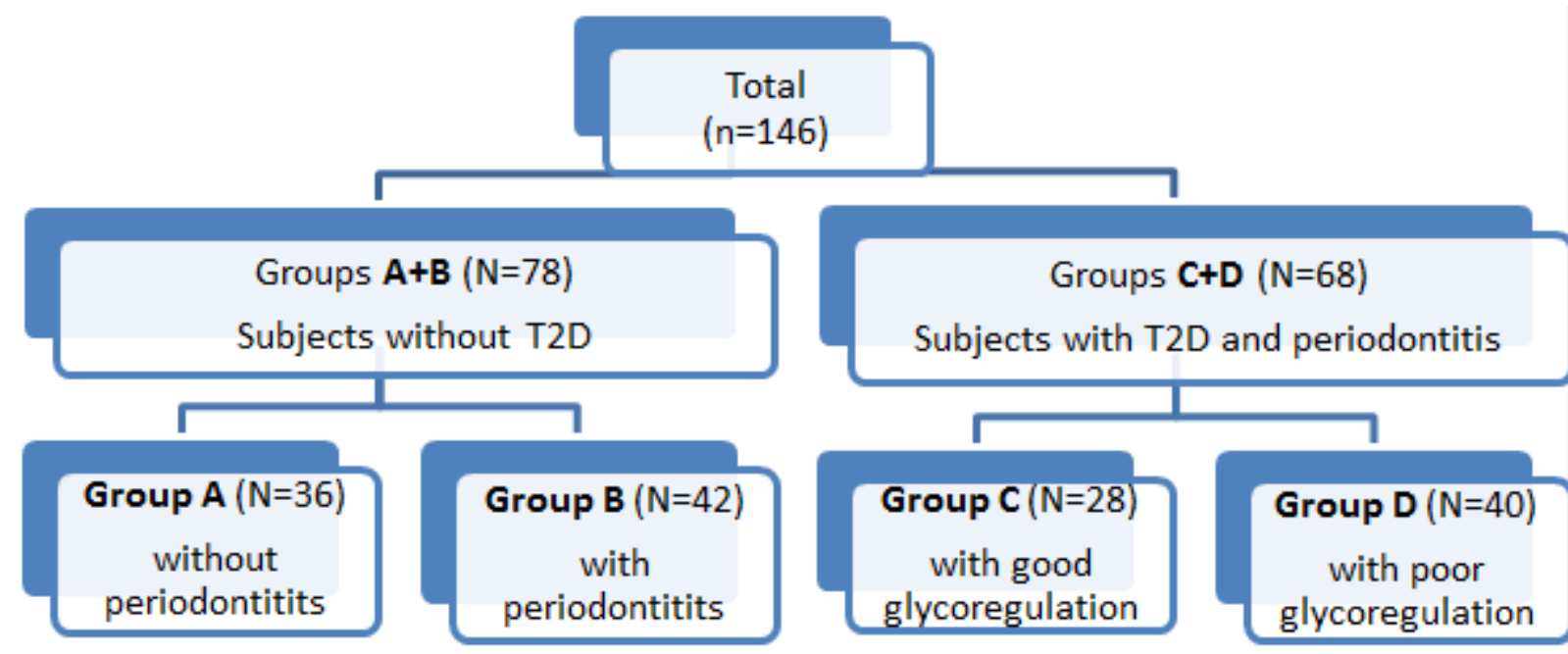

Figure 1. Study design with study groups

Cotton swab samples from tongue and subgingival samples were obtained from each patient using sterile paper points and a sterile curette as shown in Figure 2.

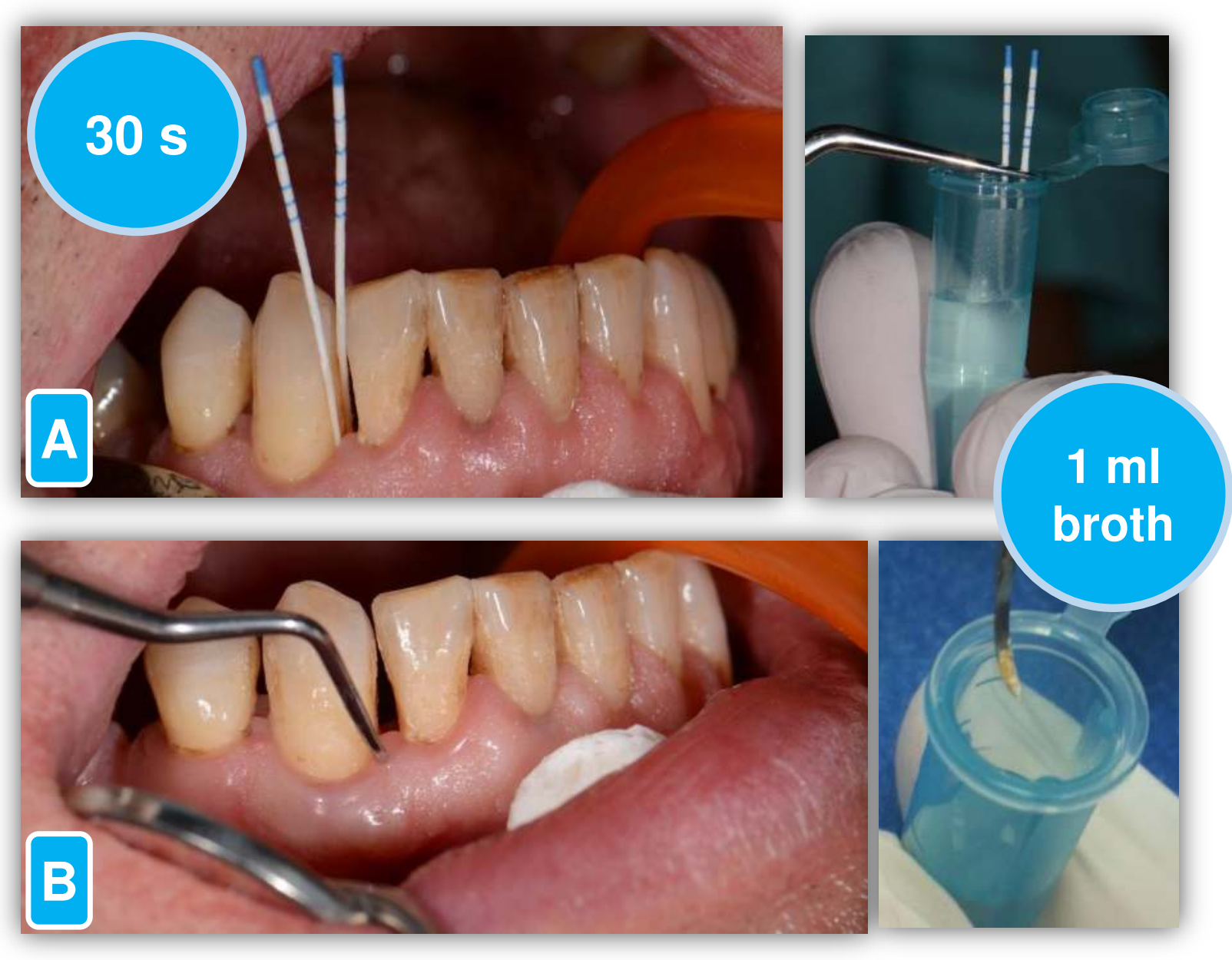

Figure 2. Subgingival sampling.

A: With 2 sterile paper points; $B$ : With sterile curette
Information about parameters that could potentially predict the presence of Candida spp. was collected. It included self-reported information about education, xerostomia, blood type, everyday intake of sweets and smoking habits. Fasting plasma glucose levels (FPG), $\mathrm{HbA1c}$, hematological parameters (RBC, Hgb, $\mathrm{HCT}, \mathrm{MCV}, \mathrm{MCH}, \mathrm{MCHC}, \mathrm{RDW}$ ) and sedimentation rate were measured

\section{Results}

Candida spp. was more prevalent in diabetics with poor glycoregulation than in other groups ( $x 2$ : $A$ vs $D, p=0.019$, $B$ vs $D, p=0.002$ and $C$ vs $D, p=0.025$ ). Subjects with T2D had a significantly higher prevalence of Candida spp. detection $(37.3 \%)$, than systematically healthy subjects $(x 2, p=0.013)$. The presence of Candida spp. in subgingival areas was $29.25 \%$ (43/146). Occurrence of Candida spp. in group D was significantly higher than in group $A(x 2, p=0.018)$, group $B\left(x^{2}\right.$, $p=0.023)$ and group $C(x 2, p=0.005)$. There were $22 / 146$ $(15.07 \%)$ cases where yeasts were not detected on the tongue, but were found in the subgingival area. Multivariate regression model showed that $\mathrm{HbA1c}$ was only the predictor of Candida spp. on the tongue and in subgingival plaque samples.

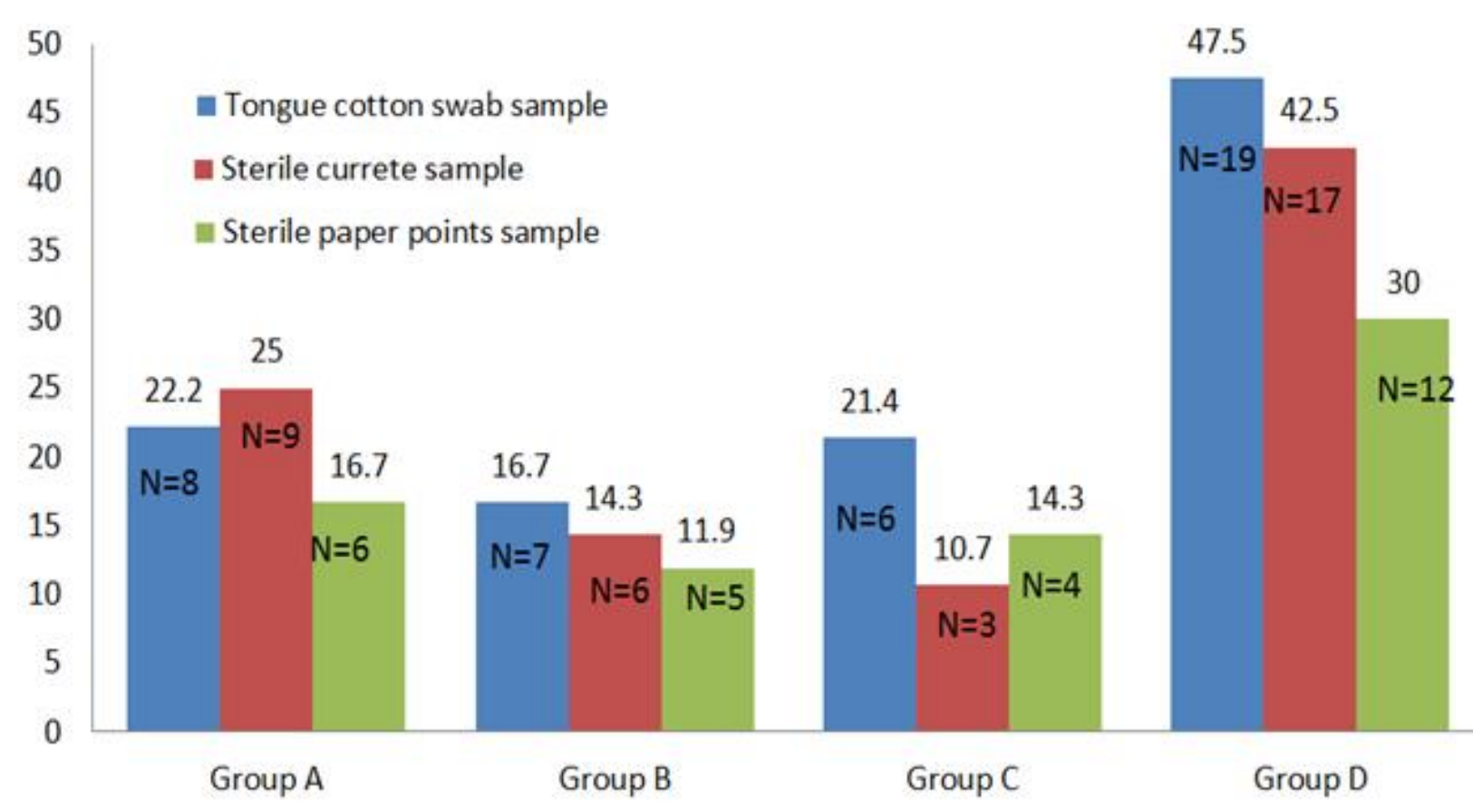

Figure 3: Frequencies of Candida spp. detection on dorsum of tongue and in subgingival areas

\section{Conclusion}

Presence of Candida spp. on the tongue and subgingival sites is higher in diabetics with poor glycoregulation and is influenced only by HbA1c serum level. High frequency of Candida spp. detection in subgingival areas (even when they are not found on the tongue) should be clarified as potential reservoir of these microorganisms.

1. Matić Petrović S, Cimbaljević M, Radunović M, Kuzmanović Pfićer J, Jotić A, Pucar A. Detection and sampling methods for isolation of Candida spp. from oral cavities in diabetics and non-diabetics. Brazilian oral research 2015;29(1): 1-7.

2. Petrović SM, Barać M, Pfićer JK, Radunović M, Jotić A, Pucar A. Presence of Different Candida Species at Denture Wearers With Type 2 Diabetes and Clinically Healthy Oral Mucosa-Pilot Study. Balkan Journal of Dental Medicine. 2018 Mar 1;22(1):15-21. 